\title{
An early prediction model for gestational diabetes mellitus based on genetic variants and clinical characteristics in China
}

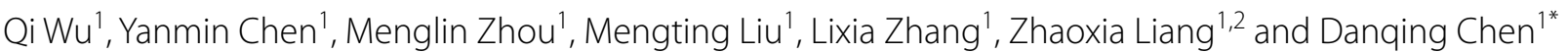

\begin{abstract}
Objectives: To evaluate the influence of genetic variants and clinical characteristics on the risk of gestational diabetes mellitus (GDM) and to construct and verify a prediction model of GDM in early pregnancy.

Methods: Four hundred seventy five women with GDM and 487 women without, as a control, were included to construct the prediction model of GDM in early pregnancy. Both groups had complete genotyping results and clinical data. They were randomly divided into a trial cohort (70\%) and a test cohort (30\%). Then, the model validation cohort, including 985 pregnant women, was used for the external validation of the GDM early pregnancy prediction model.

Results: We found maternal age, gravidity, parity, BMI and family history of diabetes were significantly associated with GDM (OR> 1; $P<0.001)$, and assisted reproduction was a critical risk factor for $G D M(O R=1.553, P=0.055)$. MTNR1B rs 10830963, C2CD4A/B rs1436953 and rs7172432, CMIP rs 16955379 were significantly correlated with the incidence of GDM (AOR> 1, $P<0.05)$. Therefore, these four genetic susceptible single nucleotide polymorphisms (SNPs) and six clinical characteristics were included in the construction of the GDM early pregnancy prediction model. In the trial cohort, a predictive model of GDM in early pregnancy was constructed, in which genetic risk score was independently associated with $\mathrm{GDM}(\mathrm{AOR}=2.061, P<0.001)$ and was the most effective predictor with the exception of family history of diabetes. The ROC-AUC of the prediction model was 0.727 (95\% Cl 0.690-0.765), and the sensitivity and specificity were $69.9 \%$ and $64.0 \%$, respectively. The predictive power was also verified in the test cohort and the validation cohort.
\end{abstract}

Conclusions: Based on the genetic variants and clinical characteristics, this study developed and verified the early pregnancy prediction model of GDM. This model can help screen out the population at high-risk for GDM in early pregnancy, and lifestyle interventions can be performed for them in a timely manner in early pregnancy.

Keywords: Gestational diabetes mellitus, Single nucleotide polymorphism, Clinical characteristics, Prediction model, Early pregnancy

\section{Introduction}

Gestational diabetes mellitus (GDM) is a common obstetric disease that affects nearly $7 \%$ of pregnant women and their offspring [1]. It is known to be associated with

\footnotetext{
*Correspondence: chendq@zju.edu.cn

1 Obstetrical Department, Women's Hospital, School of Medicine, Zhejiang University, 1 Xueshi Road, Hangzhou 310006, China

Full list of author information is available at the end of the article
}

numerous adverse perinatal outcomes, such as gestational hypertension, eclampsia, abortion, preterm delivery, macrosomia, stillbirth, and others, which complicate $3 \%$ to $25 \%$ of pregnancies [2-4]. In addition, the incidence of type 2 diabetes (T2DM) for GDM women is as high as $50 \%$ to $70 \%$ during postpartum follow-up [5], making GDM a main source of T2DM in middle-aged women. More seriously, GDM can also have an impact on the long-term health of offspring, leading to a higher risk original author(s) and the source, provide a link to the Creative Commons licence, and indicate if changes were made. The images or other third party material in this article are included in the article's Creative Commons licence, unless indicated otherwise in a credit line to the material. If material is not included in the article's Creative Commons licence and your intended use is not permitted by statutory regulation or exceeds the permitted use, you will need to obtain permission directly from the copyright holder. To view a copy of this licence, visit http://creativecommons.org/licenses/by/4.0/. The Creative Commons Public Domain Dedication waiver (http://creativeco mmons.org/publicdomain/zero/1.0/) applies to the data made available in this article, unless otherwise stated in a credit line to the data. 
of obesity, hyperlipidemia, T2DM, hypertension, brain damage and neuropsychiatric disorders through genetic susceptibility, hyperglycemia, chronic inflammation and other comprehensive factors [6].

For these reasons, early prevention and treatment of GDM is critical for ameliorating both short-term and long-term consequences for mothers and offspring. However, the oral glucose tolerance test (OGTT) is currently used in the diagnosis of GDM during the second trimester (24-28 weeks) [7], when the adverse effects of hyperglycemia on mothers and fetuses has already occurred. Unfortunately, at present there is no authoritative high-risk screening for GDM in early pregnancy. The risk of GDM is determined by both genetic susceptibility and environmental factors. GDM and its secondary T2DM are complex polygenic diseases with the following characteristics: more than one gene is involved in pathogenesis, and each gene has a different degree of action [8]. The genome-wide association study (GWAS) identified various susceptibility loci for GDM and T2DM in different population [9-12]. Some single nucleotide polymorphisms (SNPs) have been found to be associated with susceptibility to GDM in Asian population [13], such as Korean [14] and Thai [15], but the results remained inconclusive in Chinese population. In addition, previous studies also indicated that women with advanced maternal age [16], obesity [17], sedentary lifestyle [18] were more likely to develop GDM. Therefore, we intended to find GDM-associated SNPs in Chinese population and hypothesized that an early pregnancy prediction model for GDM can be constructed using genetic risk variants and clinical factors.

To test this hypothesis, we performed a case-control study to assess the combined effects of genetic information and clinical factors for the early prediction of GDM and validated its predictive values in another prospective cohort.

\section{Methods}

This study was conducted in the Women's Hospital School of Medicine Zhejiang University, including pregnant women who underwent a 75-g OGTT at 24-28 gestational weeks and consented to DNA sampling at our center. According to the recommendations from the International Association of Diabetes and Pregnancy Study Groups (IADPSG) [7], women with any blood glucose value greater than the criteria (fasting blood glucose [FBG] $5.1 \mathrm{mmol} / \mathrm{L}$, blood glucose after $1 \mathrm{~h}$ [1-h BG] $10.0 \mathrm{mmol} / \mathrm{L}$, blood glucose after $2 \mathrm{~h}$ [2-h BG] $8.5 \mathrm{mmol} / \mathrm{L}$ ) were diagnosed with GDM, while the rest formed a control group. Approval for this study was obtained from the hospital's ethics committee (IRB-20200162-R).
We designed the early pregnancy prediction model for GDM using a two-phase approach, which included development and validation. During the development phase, we performed a case-control study with 500 GDM and 500 controls to initially establish the prediction model. Because clinical characteristics were indispensable in the model construction, we randomly selected pregnant women who came to the hospital at the same time period without matching. The development cohort was randomly divided into the trial cohort (70\%) to construct the prediction model and the test cohort (30\%) to internally validate the predictive effect of the model. Moreover, we established a validation cohort with another 1000 participants to externally verify the performance of the model.

We genotyped 16 SNPs previously reported to be significantly associated with GDM or T2DM, including variants in loci known to regulate insulin secretion and function in GDM (MTNR1B, SLC30A8, CDKAL1, etc.) and in loci associated with T2DM or GDM through other potential mechanisms $(C 2 C D 4 A / B, C M I P$, etc.) (See Additional file 1: Table S1 for details of the selection). Genotyping was performed by Mulitiplex Snapshot assays, and all genotype distributions did not deviate significantly from the Hardy-Weinberg equilibrium $(P \geq 0.05)$ (Additional file 1: Table S2). In our study, of the 16 SNPs evaluated, 4 SNPs (rs10830963 in MTNR1B, rs1436953 and rs7172432 in $C 2 C D 4 A / B$, rs16955379 in $C M I P$ ) were found to be associated with the risk of GDM in at least one genetic model (Additional file 1: Tables S3 and 4), and so they were included in subsequent analyses. Pregnant women missing information for those four SNPs were excluded from the prediction model.

The clinical characteristics of early pregnancy were gathered from medical records, such as maternal age, gravidity, parity, height (self-reported at first prenatal care), pre-pregnancy weight (self-reported at first prenatal care), body mass index (BMI was calculated by weight and height), the way of conception, family history, previous medical history, and others. Pregnant women with missing or abnormal clinical information were excluded, as were those with pre-pregnancy conditions such as diabetes, hypertension, or other vital organ diseases.

Blood glucose, insulin levels and glycosylated hemoglobin (HbA1c) were tested in the biochemical laboratory of the Women's Hospital School of Medicine Zhejiang University at 24-28 gestational weeks. Homeostatic model assessments of islet $\beta$-cell function (HOMA- $\beta$ ) and insulin resistance (HOMA-IR) were calculated by FBG and fasting insulin levels.

Assuming an additive genetic model, the genotypes were scored as 0,1 , and 2 for each risk allele, and the individual effect of each SNP on the risk of GDM were evaluated by logistic regression analysis with or without 
adjustment of clinical characteristics. Multiple linear regression analysis with adjustment for maternal age, gravidity, parity, BMI, family history of diabetes and the way of conception was performed to explore the relationship between each SNP and continuous variables (e.g., blood glucose, insulin levels, HbA1c, HOMA). Bonferroni correction was used to counteract the problem of multiple comparisons. The genetic risk score (GRS) was counted to clarify the combined effect of four SNPs on the risk of GDM, which was calculated by single-SNP logistic regression analysis to better assess the genetic effect of each SNPs. The clinical characteristics between the GDM and control groups were compared by t test or $\chi^{2}$ test. The prediction model was constructed by logistic regression analysis. Receiver operating characteristic (ROC) -area under the curve (AUC) was calculated to evaluate the predictive powers. Statistical analyses were performed using SPSS version 20.0. $P<0.05$ was considered statistically significant.

\section{Results}

Inclusion of model factors in the development phase

Among Chinese pregnant women, 475 GDM and 487 controls with complete four SNPs genotype data and clinical information were included in the analysis of the development cohort (Fig. 1). In general, pregnant women with GDM had higher maternal age, gravidity, parity,
BMI than the controls, so did the rate of family history of diabetes (all $P<0.001$ ). We also noticed assisted reproduction was more likely in GDM, although it did not achieve statistical significance $(7.2 \%$ vs $10.7 \%, P=0.054)$. As expected, the blood glucose, insulin and HbA1c levels were significantly higher in GDM compared to the controls (all $P<0.001$ ). Detailed information was supplied in Additional file 1: Table S5.

We performed a univariate statistical analysis of the correlation between the clinical characteristics of early pregnancy and GDM. The results showed that maternal age, gravidity, parity, BMI, family history of diabetes were significantly associated with the increased risk of GDM $(\mathrm{OR}>1 ; P<0.001)$. Stratified analysis revealed that the risk of GDM increased with maternal age, especially in pregnant women over 40 years old whose risk of GDM was 18.79 times higher than women under 25 . Women with more than two previous pregnancies were more likely to develop GDM than women pregnant for the first time (OR 2.509, 95\% CI 1.837-3.428). In addition, overweight was a risk factor for GDM (OR 3.745, 95\% CI 2.377-5.901), while underweight was a protective factor (OR 0.684, 95\% CI 0.472-0.993). As for assisted reproduction, it also tended to increase the risk of GDM, which was borderline-significant. Thus, these six clinical characteristics were incorporated into the prediction model of GDM (Table 1).

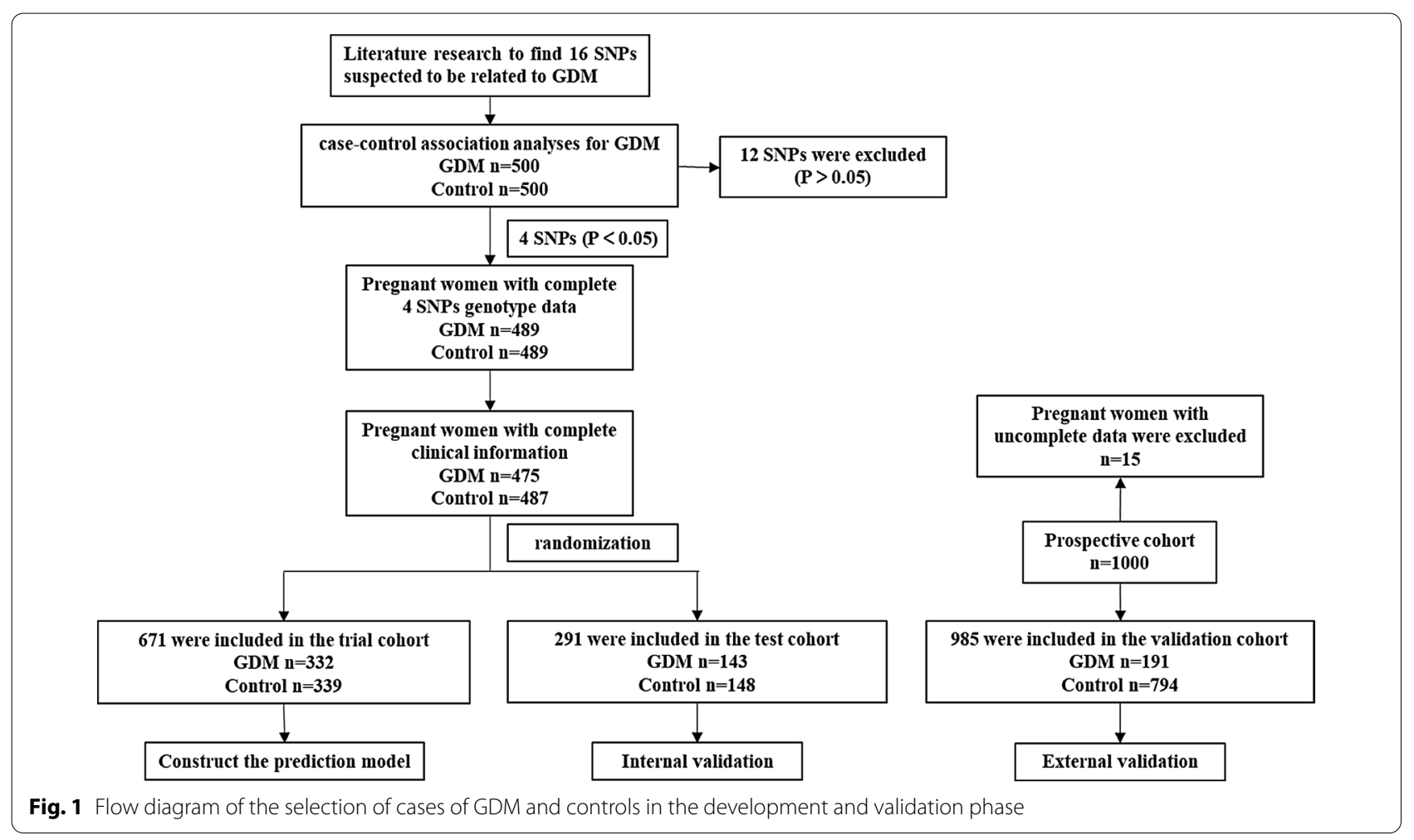


Table 1 Association of clinical characteristics with GDM in the Chinese population

\begin{tabular}{|c|c|c|c|}
\hline & The proportion of GDM ${ }^{a}$ & $\mathrm{OR}^{\mathrm{b}}(95 \% \mathrm{Cl})$ & $P$ \\
\hline Maternal age (years) & & $1.174(1.136-1.213)$ & $<0.001$ \\
\hline$<25$ & $4(15.4)$ & - & - \\
\hline $25-29$ & $91(32.7)$ & $2.676(0.896-7.996)$ & 0.078 \\
\hline $30-34$ & $190(49.9)$ & $5.471(1.850-16.177)$ & 0.002 \\
\hline $35-39$ & $149(66.5)$ & $10.927(3.634-32.855)$ & $<0.001$ \\
\hline$\geq 40$ & $41(77.4)$ & $18.792(5.414-65.230)$ & $<0.001$ \\
\hline Gravidity & & $1.397(1.248-1.565)$ & $<0.001$ \\
\hline 1 & $152(41.3)$ & - & - \\
\hline 2 & $127(44.3)$ & $1.128(0.826-1.541)$ & 0.449 \\
\hline$\geq 3$ & $196(63.8)$ & $2.509(1.837-3.428)$ & $<0.001$ \\
\hline Parity & & $1.686(1.334-2.133)$ & $<0.001$ \\
\hline Nulliparous & $218(42.1)$ & - & - \\
\hline Multiparous & $257(57.9)$ & $1.891(1.463-2.445)$ & $<0.001$ \\
\hline Pre-pregnancy BMI $\left(\mathrm{kg} / \mathrm{m}^{2}\right)$ & & $1.205(1.148-1.265)$ & $<0.001$ \\
\hline Normal $\left(18.5-24.9 \mathrm{~kg} / \mathrm{m}^{2}\right)$ & $53(37.9)$ & - & - \\
\hline Underweight $\left(<18.5 \mathrm{~kg} / \mathrm{m}^{2}\right)$ & $332(47.1)$ & $0.684(0.472-0.993)$ & $<0.001$ \\
\hline Overweight ( $\geq 25 \mathrm{~kg} / \mathrm{m}^{2}$ ) & $90(76.9)$ & $3.745(2.377-5.901)$ & $<0.001$ \\
\hline \multicolumn{4}{|l|}{ Family history of diabetes } \\
\hline No & $439(47.8)$ & - & - \\
\hline Yes & $36(81.8)$ & $4.910(2.258-10.679)$ & $<0.001$ \\
\hline \multicolumn{4}{|l|}{ Way of conception } \\
\hline Natural reproduction & $424(48.4)$ & - & - \\
\hline Assisted reproduction & $51(59.3)$ & $1.553(0.990-2.437)$ & 0.055 \\
\hline
\end{tabular}

a The proportion of GDM was expressed as number (percentage)

b Univariate logistic regression in the Chinese population

The four selected SNPs, rs10830963 in MTNR1B, rs1436953 and rs7172432 in $C 2 C D 4 A / B, \operatorname{rs} 16955379$ in $C M I P$ were significantly related to GDM with or without adjustment for clinical information. Among them, rs10830963 had the strongest relationship with GDM (adjusted OR 1.387, 95\% CI 1.136-1.694), while rs1436953, rs7172432, rs16955379 were found to have a 1.257-fold, 1.308-fold and 1.291-fold increased risk of GDM, respectively (Table 2). Collinearity diagnosis suggested that there was no collinearity among the four
SNPs (Additional file 1: Table S6). Moreover, the $G$ allele of rs10830963 was correlated with elevated 1-h OGTT BG $(\beta=0.286, P=0.002)$ and HOMA-IR $(\beta=0.162$, $P=0.017)$, while the $T$ allele of rs1436953 was associated with increased 2-h OGTT BG $(\beta=0.222, P=0.010)$ and HOMA-IR $(\beta=0.175, P=0.011)$. We also observed the association of rs7172432 with higher 1-h OGTT BG $(\beta=0.214, P=0.025), 2$-h OGTT BG $(\beta=0.242$, $P=0.005)$, HbA1c $(\beta=0.036, P=0.038)$, HOMA-IR $(\beta=0.157, P=0.020)$. The $C$ allele of rs16955379 also

Table 2 Single-SNP association analysis of GDM

\begin{tabular}{|c|c|c|c|c|c|c|c|c|c|}
\hline Closest gene & SNP & $A / a$ & $\begin{array}{l}\text { Control } \\
\text { AA/Aa/aa }\end{array}$ & $\begin{array}{l}\text { GDM } \\
\text { AA/Aa/aa }\end{array}$ & OR $(95 \% \mathrm{Cl})$ & $P$ & $\mathrm{aOR}^{\mathrm{a}}(95 \% \mathrm{Cl})$ & $\beta^{b}$ & $P$ \\
\hline MTNR1B & rs10830963 & $\mathrm{C} / \underline{\mathrm{G}}$ & $161 / 239 / 87$ & $118 / 246 / 111$ & $1.327(1.105-1.592)$ & 0.002 & $1.387(1.136-1.694)$ & 0.327 & 0.001 \\
\hline$C 2 C D 4 A / B$ & rs1436953 & $\mathrm{C} / \underline{\mathbf{T}}$ & $236 / 205 / 46$ & $194 / 218 / 63$ & $1.292(1.068-1.562)$ & 0.008 & $1.257(1.021-1.548)$ & 0.229 & 0.031 \\
\hline$C 2 C D 4 A / B$ & rs7172432 & $\mathrm{A} / \underline{\mathbf{G}}$ & $203 / 227 / 57$ & $161 / 242 / 72$ & $1.283(1.062-1.551)$ & 0.010 & $1.308(1.061-1.611)$ & 0.268 & 0.012 \\
\hline$C M I P$ & rs16955379 & $\underline{C} / T$ & 250/189/48 & $268 / 175 / 32$ & $1.220(1.001-1.486)$ & 0.048 & $1.291(1.039-1.605)$ & 0.256 & 0.021 \\
\hline
\end{tabular}

$\mathrm{A} / \mathrm{a}$, major allele/minor allele; Risk allele was underlined and in bold

${ }^{a}$ The analysis was adjusted for maternal age, gravidity, parity, BMI, family history of diabetes and way of conception

${ }^{\mathrm{b}}$ Regression coefficients in the multivariable logistic regression analysis 


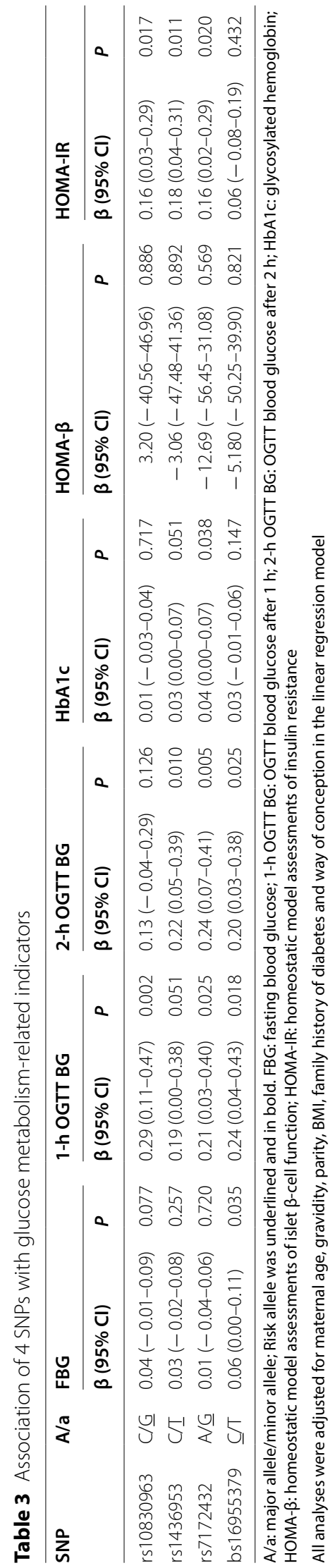


had the similar effect on FBG $(\beta=0.055, P=0.035)$, 1 -h OGTT BG $(\beta=0.236, P=0.018)$ and 2-h OGTT BG $(\beta=0.201, P=0.025)$. Nevertheless, the difference was no longer significant after Bonferroni correction, except for the $G$ allele of rs10830963 and 1-h OGTT BG, and the G allele of rs7172732 and 2-h OGTT BG (Table 3).

\section{Model construction and internal validation in the development phase}

According to the above results, we planned to establish the GDM prediction model in the trial cohort, including maternal age, gravidity, parity, pre-pregnancy BMI, family history of diabetes, the way of conception and GRS that was calculated to clarify the combined effect of the four SNPs on the risk of GDM. In the model, we found that GRS was independently correlated with GDM (aOR 2.061, 95\% CI 1.382-3.073), which was the most effective predictor, with the exception of family history of diabetes (aOR 4.133, 95\% CI 1.613-10.585) (Table 4). The ROC-AUC of the prediction model with GRS and clinical characteristics was 0.727 (95\% CI $0.690-0.765$ ), and the sensitivity and specificity were $69.9 \%$ and $64.0 \%$, respectively. In addition, the Hosmer-Lemeshow test showed that the model had a good calibration ability $\left(x^{2}=5.141\right.$, $P=0.742$ ) (Fig. 2).

We then evaluated the predictive power of the constructed model in the test cohort. The ROC-AUC in the test cohort was 0.776 (95\% CI 0.722-0.830), and the sensitivity and specificity were respectively $71.3 \%$ and 75.0\%. The Hosmer-Lemeshow test showed that there was no significant difference between the fitted values and the observed values among GDM groups $\left(X^{2}=6.637\right.$, $P=0.576$ ) (Additional file 1: Fig. S1A).

\section{External validation in the validation phase}

In the analysis of the validation cohort, there were 985 participants with complete four SNP-genotype data and clinical information, including 191 GDM and 794 controls, with a $19.4 \%$ incidence of GDM (Fig. 1). The clinical characteristics were similar to those found in the development cohort (Additional file 1: Table S5). The prediction model was externally verified in the validation cohort, whose ROC-AUC was 0.620 (95\% CI $0.573-0.667$ ) with a sensitivity of $52.4 \%$ and a specificity of $68.8 \%$. The Hosmer-Lemeshow test also showed the good calibration ability of the GDM prediction model $\left(\chi^{2}=10.741, P=0.217\right)$ (Additional file 1: Fig. S1B).

\section{Discussion}

Based on genetic variants and clinical characteristics in early pregnancy, this study constructed a prediction model of GDM in a two-phase approach. In this model, a total of 4 SNPs and 6 basic clinical features were included, which were closely related to the risk of GDM in Chinese pregnant women. The SNPs can be determined by genotyping from peripheral blood of pregnant women in early pregnancy. Clinical features can be simply obtained from the medical records in the first prenatal examination. By inputting these data, the model can screen out women at high risk of GDM early in pregnancy in order to facilitate a timely intervention.

In this study, we observed that the $G$ allele of rs10830963 in MTNR1B was significantly associated with the risk of GDM, which increased 1.387 times for each additional risk allele. Previous studies found similar results: polymorphism at rs10830963 was a specific genetic factor for GDM $[19,20]$. In fact, MTNR1B was strongly expressed in islet $\beta$ cells and maintained glucose homeostasis by regulating insulin release [21]. Our results suggested that the $G$ allele of rs 10830963 was independently associated with increased 1-h OGTT BG and insulin resistance, which also supported the possibility of this mechanism from an epidemiological perspective. Although the molecular mechanism of $C 2 C D 4 A / B$ in regulating glucose homeostasis has not been wellcharacterized, the association of risk alleles in $C 2 C D 4 A / B$ with T2DM has been repeatedly demonstrated in several studies [22-24]. In our study, two loci of $C 2 C D 4 A / B$ were first identified to be associated with GDM in Chinese pregnant women. Women with $T$ alleles in rs1436953 and $G$ alleles in rs7172432 were more susceptible to GDM. Furthermore, our study on relationship between $C 2 C D 4 A / B$ variants and glucose metabolism-related indicators suggested that $C 2 C D 4 A / B$ might be related to islet dysfunction in GDM, which was not surprising given the similarities in the pathogenesis and epidemiology of T2DM and GDM. Interestingly, this study also found for the first time a significant association between CMIP rs16955379 and GDM. Previous studies suggested that $C M I P$ was associated with lipid metabolism, and its rs16955379 variant was linked to lipid metabolism disorders [25], which might thus increase the risk of T2DM [26]. Cho et al. [11] found $C$ alleles in rs16955379 was a risk factor for T2DM in East Asian populations. More importantly, our study also showed that the $C$ allele of rs16955379 could increase the levels of FBG, 1-h OGTT BG and 2-h OGTT BG in pregnant women, which concurred with previous studies [27].

In addition to genetic variants, clinical characteristics also played a significant role in the incidence of GDM. Advanced maternal age has always been a high risk factor for GDM. Khali et al. [28] found that the incidence of GDM was positively correlated with maternal age and reached a peak at the age of 40 . We also found pregnant women with multiple gravidities or parities were more likely to suffer from GDM, which 
Table 4 Prediction model for GDM constructed in the trial cohort

\begin{tabular}{|c|c|c|c|c|c|c|}
\hline Model & $\mathrm{OR}^{\mathrm{a}}$ & $P$ & $a O R^{b}$ & $\beta$ & Wals & $P$ \\
\hline GRS & $1.855(1.287-2.674)$ & 0.001 & $2.061(1.382-3.073)$ & 0.723 & 12.598 & $<0.001$ \\
\hline Maternal age & 1.150 (1.107-1.194) & $<0.001$ & 1.133 (1.082-1.187) & 0.125 & 27.879 & $<0.001$ \\
\hline Gravidity & $1.364(1.194-1.559)$ & $<0.001$ & 1.089 (0.896-1.323) & 0.085 & 0.734 & 0.392 \\
\hline Parity & $1.712(1.291-2.270)$ & $<0.001$ & $0.957(0.629-1.457)$ & -0.044 & 0.042 & 0.838 \\
\hline Pre-pregnancy BMI & $1.198(1.131-1.269)$ & $<0.001$ & $1.167(1.100-1.239)$ & 0.155 & 25.936 & $<0.001$ \\
\hline Family history of diabetes & $4.520(1.829-11.165)$ & 0.001 & $4.133(1.613-10.585)$ & 1.419 & 8.743 & 0.003 \\
\hline Way of conception & $1.596(0.930-2.740)$ & 0.090 & 1.140 (0.619-2.098) & 0.131 & 0.176 & 0.675 \\
\hline
\end{tabular}

a Univariate logistic regression in the trial cohort

${ }^{\mathrm{b}}$ The prediction model was constructed by multivariate logistic regression

has been confirmed by previous studies [29, 30]. Further, higher pre-pregnancy BMI was also a common risk factor for GDM. Obesity not only greatly increased the risk of GDM, but also made fetal congenital abnormalities, preterm delivery and even death more likely to occur [31]. Family history of diabetes was a clinical characteristic most strongly associated with GDM, and it increased the risk of GDM by more than 4 times in our study. This result was also reported by Harder et al. [32], and they found the influence of maternal diabetes on pregnant women was stronger than that of paternal diabetes. Besides, although no significant effect of assisted reproduction on the incidence of GDM was found in our study, it was still included in the construction of the prediction model. The reason for this was that current evidence indicated assisted reproduction could increase the risk of GDM [33, 34], and the experience of clinicians has also suggested pregnant women with assisted reproduction need more strict management to prevent GDM than those who conceive naturally.

In summary, we used the trial cohort to construct a prediction model of GDM in early pregnancy based on genetic variants and clinical characteristics. The prediction efficiency reached 0.727 with sensitivity and specificity of $69.9 \%$ and $64.0 \%$, respectively. We then used the test cohort to primarily verify the model and found that the prediction model still had good modeldiscrimination ability. However, considering that internal validation often overestimates prediction accuracy, and we needed to determine whether the prediction model is valid for clinical applications, we prospectively collected validation cohort to conduct an external validation of the prediction model. The results of external validation showed that the model prediction efficiency did not become more ideal, but it still had a certain capability for model discrimination. Although model sensitivity was reduced, the model specificity remained excellent, reaching $68.8 \%$. This means that the model can effectively exclude most non-GDM patients, and the others who may at high risk of GDM can be reduced by timely and effective lifestyle intervention and management.

As a pregnancy complication that seriously threatens the health of both mother and child, screening and diagnosis methods for GDM have long been a focus of research. All involved professional organizations recommend that pregnant women should take the OGTT at 24-28 gestational weeks as the gold standard for diagnosing GDM [35], but undertaking it is not advised for pregnant women in the early stage of pregnancy. This may be due to the fact that abnormal blood glucose detected thus may have existed before pregnancy and therefore cannot be clearly diagnosed as typical GDM that develops during pregnancy [36]. Initially, the IADPSG also proposed to use the FBG threshold of $>92 \mathrm{mg} \%$ as the diagnostic method for GDM before 24 gestational weeks. However, this was ultimately removed from the recommendation due to the lack of evidence for a convincing threshold in early pregnancy [37]. Instead of focusing on OGTT and blood glucose screening, we innovatively established an early pregnancy prediction model using genetic variants and clinical characteristics, which could be used to screen pregnant women at high risk of GDM in early pregnancy. It was the first advantage of our study. Many researchers have explored GDM prediction models in the past, but most of them were based on clinical features. Pan et al. [38] explored the combined predictive effect of pre-pregnancy BMI and first-trimester FBG on GDM. Sweetin et al. [39] developed a prediction model for early pregnancy on the basis of maternal lipid metabolites and clinical data. These studies ignored the important role of genetic factors in the incidence of GDM. Kawai et al. [40] explored the relationship between common T2DM risk SNPs and GDM, but their results were not prospectively verified in a new pregnant-women population, as it was in our study. The rigorous process of model predictor inclusion, construction and validation of the model was 
(A) ROC for GDM prediction model.

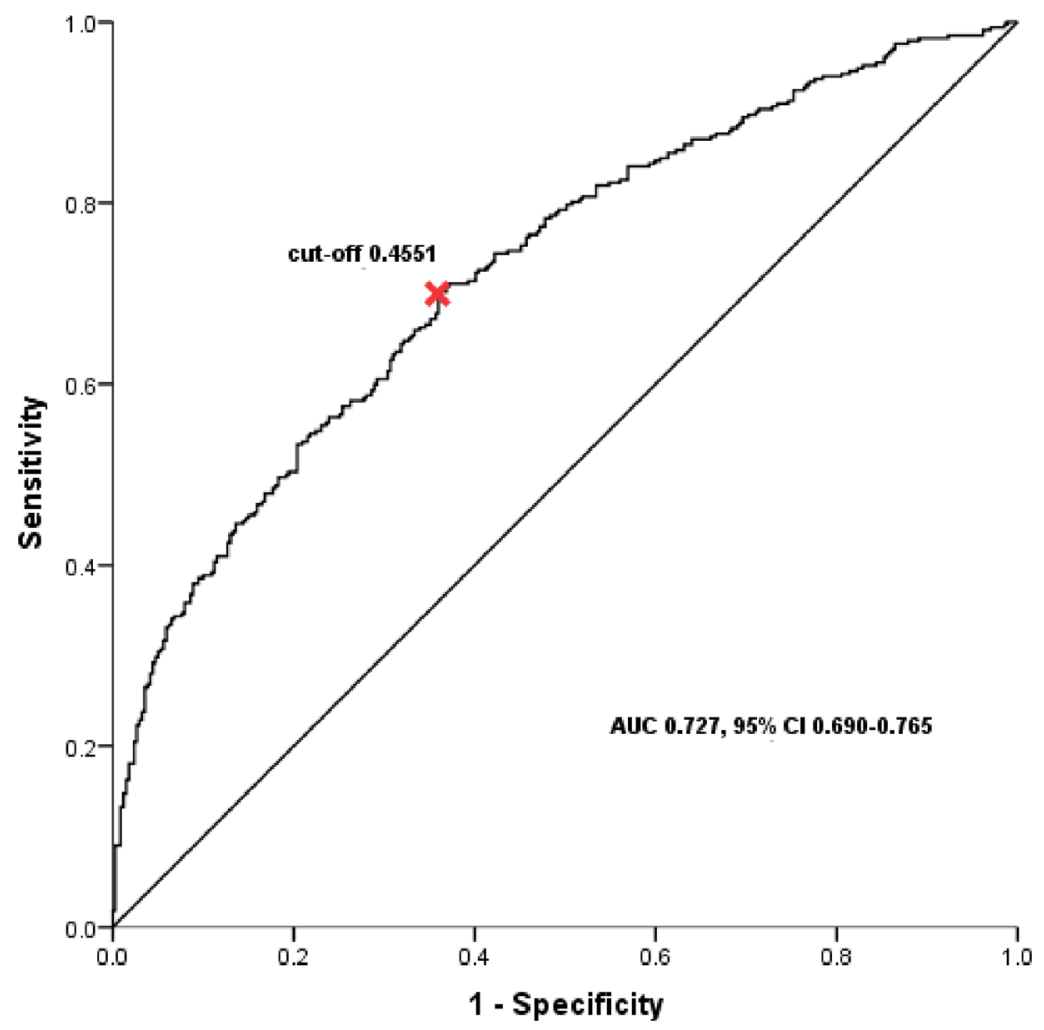

(B) Curve-fitting performance of the GDM prediction model.

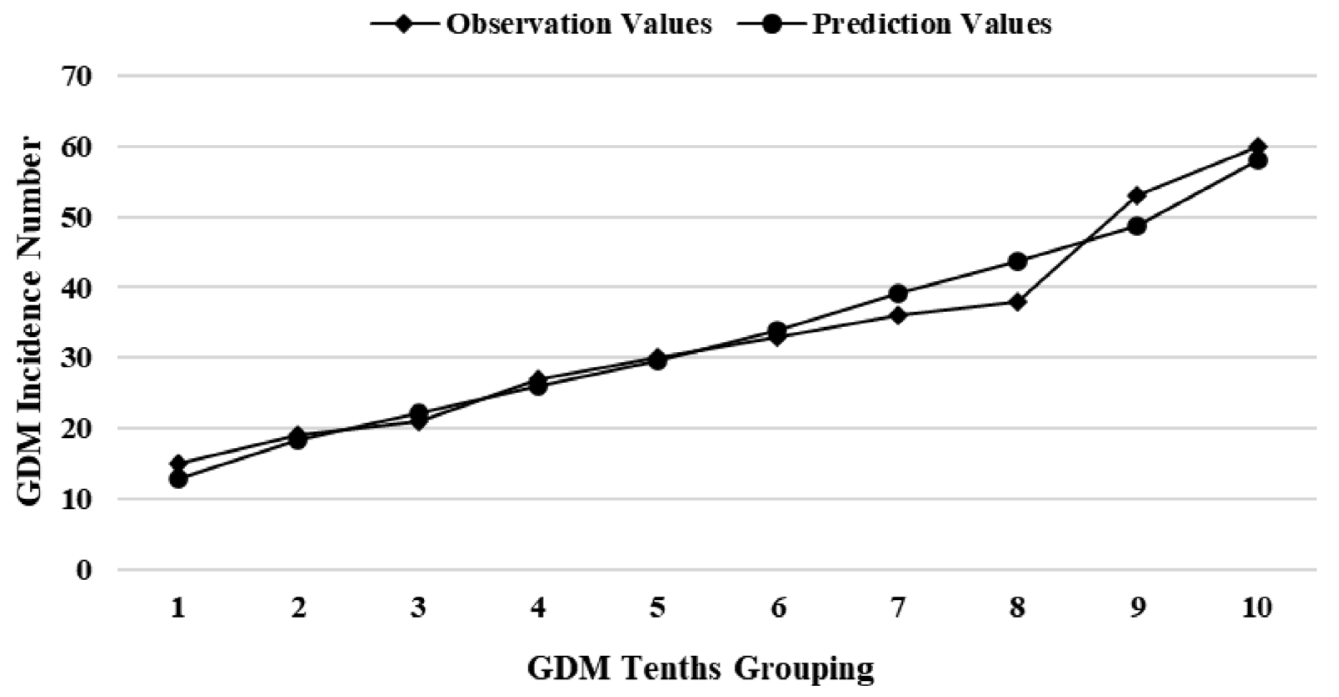

Fig. 2 The performance of the GDM prediction model with GRS and clinical characteristics

the second advantage of this study. Because GDM was a complex polygenic disease, we used GRS to comprehensively evaluate the combined effect of four SNPs on the risk of GDM. The results showed that GRS was strongly associated with an increased risk of GDM and was one of the most effective predictors in the prediction model. It was worth noting that we did not simply calculate the GRS by adding up each risk allele, but weighted each SNP 
according to its own correlation with GDM, so as to combine the genetic susceptibility of each SNPs more effectively. While focusing on the combined genetic effect of GDM, we did not ignore the specificity of each SNPs, which was also one of the advantages.

There were still some limitations. First, this was a regional single-center study from a large specialized hospital, whose population had a higher overall risk and could only represent pregnant women in the surrounding area of the hospital. Thus, it might not be fully applicable to all the population. However, all participants in our study were strictly grouped and analyzed to ensure the reliability of the research results. Second, this kind of study also leaded to a small sample size, which might affect its accuracy and reliability to some extent. Largescale multicenter studies need to be performed to further verify the prediction model for GDM. Third, although we adjusted gravidity and parity as confounding factors in the analysis of GDM genetic susceptibility, we could not ignore the possibility that normal control pregnant women would develop GDM in future pregnancies, which might lead to grouping error and an underestimation for the effect of genetic factors on the risk of GDM.

In conclusion, an early pregnancy prediction model of GDM based on genetic variants and clinical characteristics was developed and verified in our study. This prediction model can screen in the first trimester for pregnant women at high risk for GDM, allowing physicians to start lifestyle and dietary interventions early so that women can maintain normal blood glucose levels during pregnancy. It is expected to fundamentally reduce the incidence of GDM and improve the quality of the newborn population.

\section{Supplementary Information}

The online version contains supplementary material available at https://doi. org/10.1186/s13098-022-00788-y.

Additional file 1. Additional tables and figures.

\section{Acknowledgements}

We specially thank Chaolu Chen and Shuaiying Zhu at Women's Hospital School of Medicine Zhejiang University for their support in this study.

\section{Authors' contributions \\ QW designed the study, analyzed and interpreted data, wrote and edited the manuscript. YMC designed the study, collected and collated samples. MLZ selected statistical methodology, validated the data. MTL collected and col- lated samples, edited the manuscript. LXZ and ZXL reviewed and edited the manuscript. DQC conceived the study and edited the manuscript. All authors read and approved the final manuscript.}

\section{Funding}

This work was supported by the Key Project of Science and Technology Department of Zhejiang Province under Grant 2018C03010, National Natural Science Foundation of China under Grant 81873839, National Natural Science Foundation of China under Grant 81974234.

\section{Data availability}

Some or all datasets generated during and/or analyzed during the current study are not publicly available but are available from the corresponding author on reasonable request.

\section{Declarations}

\section{Ethics approval and consent to participate}

Approval for this study was obtained from the hospital's ethics committee of Women's Hospital School of Medicine Zhejiang University (IRB-20200162-R).

\section{Consent for publication}

All authors have agreed to submit this manuscript for publication.

\section{Competing interests}

The authors declare that there is no duality of interest associated with this manuscript.

\section{Author details}

'Obstetrical Department, Women's Hospital, School of Medicine, Zhejiang University, 1 Xueshi Road, Hangzhou 310006, China. ${ }^{2}$ Department of Epidemiology, School of Public Health and Tropical Medicine, Tulane University, New Orleans, Los Angeles, United States of America.

Received: 19 November 2021 Accepted: 6 January 2022

Published online: 24 January 2022

\section{References}

1. American Diabetes Association. 2. Classification and diagnosis of diabetes: standards of medical care in diabetes-2019. Diabetes care. 2019;42(Suppl 1):S13-s28.

2. Catalano PM, McIntyre HD, Cruickshank JK, McCance DR, Dyer AR, Metzger BE, Lowe LP, Trimble ER, Coustan DR, Hadden DR, et al. The hyperglycemia and adverse pregnancy outcome study: associations of GDM and obesity with pregnancy outcomes. Diabetes Care. 2012;35(4):780-6.

3. Billionnet C, Mitanchez D, Weill A, Nizard J, Alla F, Hartemann A, Jacqueminet S. Gestational diabetes and adverse perinatal outcomes from 716,152 births in France in 2012. Diabetologia. 2017;60(4):636-44.

4. Trujillo J, Vigo A, Duncan BB, Falavigna M, Wendland EM, Campos MA, Schmidt MI. Impact of the International Association of Diabetes and Pregnancy Study Groups criteria for gestational diabetes. Diabetes Res Clin Pract. 2015;108(2):288-95

5. Buchanan TA, Xiang AH, Page KA. Gestational diabetes mellitus: risks and management during and after pregnancy. Nat Rev Endocrinol. 2012;8(11):639-49.

6. Damm P, Houshmand-Oeregaard A, Kelstrup L, Lauenborg J, Mathiesen ER, Clausen TD. Gestational diabetes mellitus and long-term consequences for mother and offspring: a view from Denmark. Diabetologia. 2016;59(7):1396-9.

7. Metzger BE, Gabbe SG, Persson B, Buchanan TA, Catalano PA, Damm P, Dyer AR, Leiva A, Hod M, Kitzmiler JL, et al. International association of diabetes and pregnancy study groups recommendations on the diagnosis and classification of hyperglycemia in pregnancy. Diabetes Care. 2010;33(3):676-82.

8. Kwak SH, Jang HC, Park KS. Finding genetic risk factors of gestational diabetes. Genomics Inform. 2012;10(4):239-43.

9. Zhang C, Bao W, Rong Y, Yang H, Bowers K, Yeung E, Kiely M. Genetic variants and the risk of gestational diabetes mellitus: a systematic review. Hum Reprod Update. 2013;19(4):376-90.

10. Kwak SH, Kim SH, Cho YM, Go MJ, Cho YS, Choi SH, Moon MK, Jung HS, Shin HD, Kang HM, et al. A genome-wide association study of gestational diabetes mellitus in Korean women. Diabetes. 2012;61 (2):531-41.

11. Cho YS, Chen CH, Hu C, Long J, Ong RT, Sim X, Takeuchi F, Wu Y, Go MJ, Yamauchi T, et al. Meta-analysis of genome-wide association studies identifies eight new loci for type 2 diabetes in east Asians. Nat Genet. 2011;44(1):67-72. 
12. Kong Y, Sharma RB, Ly S, Stamateris RE, Jesdale WM, Alonso LC. CDKN2A/B T2D Genome-Wide Association Study Risk SNPs impact locus gene expression and proliferation in human islets. Diabetes. 2018;67(5):872-84.

13. Bai Y, Tang L, Li L, Li L. The roles of ADIPOQ rs 266729 and MTNR1B rs10830963 polymorphisms in patients with gestational diabetes mellitus: a meta-analysis. Gene. 2020;730:144302.

14. Cho YM, Kim TH, Lim S, Choi SH, Shin HD, Lee HK, Park KS, Jang HC. Type 2 diabetes-associated genetic variants discovered in the recent genomewide association studies are related to gestational diabetes mellitus in the Korean population. Diabetologia. 2009;52(2):253-61.

15. Plengvidhya N, Chanprasert C, Chongjaroen N, Yenchitsomanus PT, Homsanit M, Tangjittipokin W. Impact of KCNQ1, CDKN2A/2B, CDKAL1, HHEX, MTNR1B, SLC30A8, TCF7L2, and UBE2E2 on risk of developing type 2 diabetes in Thai population. BMC Med Genet. 2018;19(1):93.

16. Kennelly MA, McAuliffe FM. Prediction and prevention of gestational diabetes: an update of recent literature. Eur J Obstet Gynecol Reprod Biol. 2016;202:92-8.

17. Fukushima $Y$, Kurose $S$, Shinno $H$, Thi Thu HC, Takao N, Tsutsumi $H$ Hasegawa T, Nakajima T, Kimura Y. Effects of body weight reduction on serum irisin and metabolic parameters in obese subjects. Diabetes Metab J. 2016;40(5):386-95.

18. Giudici KV, Martini LA. Comparison between body mass index and a body shape index with adiponectin/leptin ratio and markers of glucose metabolism among adolescents. Ann Hum Biol. 2017:44(6):489-94.

19. Alharbi KK, Al-Sulaiman AM, Shedaid KMB, Al-Shangiti AM, Marie M, AlSheikh YA, Ali Khan I. MTNR1B genetic polymorphisms as risk factors for gestational diabetes mellitus: a case-control study in a single tertiary care center. Ann Saudi Med. 2019;39(5):309-18.

20. Zhang Y, Sun CM, Hu XQ, Zhao Y. Relationship between melatonin receptor $1 \mathrm{~B}$ and insulin receptor substrate 1 polymorphisms with gestational diabetes mellitus: a systematic review and meta-analysis. Sci Rep. 2014;4:6113.

21. Bonnefond A, Froguel P. Disentangling the role of melatonin and its receptor MTNR1B in type 2 diabetes: still a long way to go? Curr DiabRep. 2017;17(12):122

22. Cui B, Zhu X, Xu M, Guo T, Zhu D, Chen G, Li X, Xu L, Bi Y, Chen Y, et al. A genome-wide association study confirms previously reported loci for type 2 diabetes in Han Chinese. PloS ONE. 2011;6(7):e22353.

23. Zheng JS, Li K, Huang T, Chen Y, Xie H, Xu D, Sun J, Li D. Genetic risk score of nine type 2 diabetes risk variants that interact with erythrocyte phospholipid alpha-linolenic acid for type 2 diabetes in Chinese Hans: a case-control study. Nutrients. 2017;9(4):376.

24. Imamura M, Shigemizu D, Tsunoda T, Iwata M, Maegawa $H$, Watada $H$, Hirose H, Tanaka Y, Tobe K, Kaku K, et al. Assessing the clinical utility of a genetic risk score constructed using 49 susceptibility alleles for type 2 diabetes in a Japanese population. J Clin Endocrinol Metab. 2013:98(10):E1667-1673.

25. Mo MQ, Pan L, Lu QM, Li QL, Liao YH. The association of the CMIP rs16955379 polymorphism with dyslipidemia and the clinicopathological features of IgA nephropathy. Int J Clin Exp Pathol. 2018;11(10):5008-23.

26. Cao Y, Wang T, Wu Y, Juan J, Qin X, Tang X, Wu T, Hu Y. Opposite genetic effects of CMIP polymorphisms on the risk of type 2 diabetes and obesity: a family-based study in China. Int J Mol Sci. 2018;19(4):1011.

27. Sakai K, Imamura M, Tanaka Y, Iwata M, Hirose H, Kaku K, Maegawa H, Watada H, Tobe K, Kashiwagi A, et al. Replication study for the association of 9 East Asian GWAS-derived loci with susceptibility to type 2 diabetes in a Japanese population. PloS ONE. 2013;8(9):e76317.

28. Khalil A, Syngelaki A, Maiz N, Zinevich Y, Nicolaides KH. Maternal age and adverse pregnancy outcome: a cohort study. Ultrasound obstet. 2013:42(6):634-43.

29. Lee KW, Ching SM, Ramachandran V, Yee A, Hoo FK, Chia YC, Wan Sulaiman WA, Suppiah S, Mohamed MH, Veettil SK. Prevalence and risk factors of gestational diabetes mellitus in Asia: a systematic review and meta-analysis. BMC Pregnancy Childbirth. 2018;18(1):494.

30. Chen P, Wang S, Ji J, Ge A, Chen C, Zhu Y, Xie N, Wang Y. Risk factors and management of gestational diabetes. Cell Biochem Biophys. 2015;71(2):689-94.

31. Girchenko P, Tuovinen S, Lahti-Pulkkinen M, Lahti J, Savolainen K, Heinonen K, Pyhälä R, Reynolds RM, Hämäläinen E, Villa PM, et al. Maternal early pregnancy obesity and related pregnancy and pre-pregnancy disorders: associations with child developmental milestones in the prospective PREDO Study. Int J Obes. 2018;42(5):995-1007.

32. Harder T, Plagemann A. A role for gestational diabetes in the excess maternal transmission of type 2 diabetes? Diabetes Care. 2000;23(3):431-2.

33. Lei LL, Lan YL, Wang SY, Feng W, Zhai ZJ. Perinatal complications and livebirth outcomes following assisted reproductive technology: a retrospective cohort study. Chin Med J. 2019;132(20):2408-16.

34. Jie Z, Yiling D, Ling Y. Association of assisted reproductive technology with adverse pregnancy outcomes. Iran J Reprod Med. 2015;13(3):169-80.

35. Gupta Y, Kalra B, Baruah MP, Singla R, Kalra S. Updated guidelines on screening for gestational diabetes. Int J Women's Health. 2015;7:539-50.

36. Diagnosis and classification of diabetes mellitus. Diabetes Care. 2014;37(Suppl 1):S81-90.

37. Mclntyre HD, Sacks DA, Barbour LA, Feig DS, Catalano PM, Damm P, McElduff A. Issues with the diagnosis and classification of hyperglycemia in early pregnancy. Diabetes Care. 2016;39(1):53-4.

38. Pan Y, Hu J, Zhong S. The joint prediction model of pBMI and eFBG in predicting gestational diabetes mellitus. J Int Med Res. 2020:48(4):300060519889199.

39. Sweeting AN, Wong J, Appelblom H, Ross GP, Kouru H, Williams PF, Sairanen $M$, Hyett JA. A novel early pregnancy risk prediction model for gestational diabetes mellitus. Fetal Diagn Ther. 2019;45(2):76-84.

40. Kawai VK, Levinson RT, Adefurin A, Kurnik D, Collier SP, Conway D, Stein CM. A genetic risk score that includes common type 2 diabetes risk variants is associated with gestational diabetes. Clin Endocrinol. 2017:87(2):149-55.

\section{Publisher's Note}

Springer Nature remains neutral with regard to jurisdictional claims in published maps and institutional affiliations.

Ready to submit your research? Choose BMC and benefit from

- fast, convenient online submission

- thorough peer review by experienced researchers in your field

- rapid publication on acceptance

- support for research data, including large and complex data types

- gold Open Access which fosters wider collaboration and increased citations

- maximum visibility for your research: over 100M website views per year

At BMC, research is always in progress.

Learn more biomedcentral.com/submissions 